Supporting Information for:

\title{
Optical Interference Enhances Nonlinear Spectroscopic Sensitivity: When Light Gives You Lemons, Model Lemonade
}

\author{
Patrick M. Kearns, Daniel B. O'Brien, and Aaron M. Massari* \\ Department of Chemistry, University of Minnesota - Twin Cities
}

207 Pleasant St SE, Minneapolis, MN 55455

*Corresponding author. Email: massari@umn.edu 


\section{Experimental details of the VSFG measurements}

\section{Sample preparation}

VSFG data were collected on $8 \mathrm{~nm}$ thick PTCDI OFET devices in the bottom gate bottom contact geometry with a $300 \mathrm{~nm} \mathrm{SiO}$ gate dielectric. Substrates were prepared by photolithographic patterning of source and drain electrodes on silicon wafers purchased with the thermally grown and polished oxide dielectric (University Wafer). These were cleaned following the RCA protocol detailed elsewhere. ${ }^{1}$ The PTCDI was vapor deposited through a shadow mask onto the transistor channel with the substrate held at $30^{\circ} \mathrm{C}$ and post annealed at $110^{\circ} \mathrm{C}$ in a homebuilt vapor deposition chamber. Transistor channel dimensions were $1 \times 1 \mathrm{~mm}$. The capacitance of these devices was measured to be $1.19 \times 10^{-8} \mathrm{~F} / \mathrm{cm}^{2}$.

\section{VSFG spectroscopy}

The VSFG instrument setup has been described previously. ${ }^{2}$ Briefly, broadband infrared light centered at $\sim 1680 \mathrm{~cm}^{-1}$ with $175 \mathrm{~cm}^{-1}$ bandwidth FWHM was overlapped in space and time with a narrowband visible pulse centered at $800 \mathrm{~nm}$ with $1 \mathrm{~nm}$ FWHM. The mid-IR light was created by difference frequency mixing of the near-IR signal and idler beams from an optical parametric amplifier pumped at $1 \mathrm{kHz}$ by $0.5 \mathrm{~W}$ from a Ti:Sapph regenerative amplifier. The narrowband visible pulse was created by amplifying $0.3 \mathrm{~W}$ of uncompressed regen output in a Ti:Sapph multipass amplifier. The output was filtered to approximately $3 \mathrm{~nm}$ prior to multipass amplification and then further narrowed in a $4 \mathrm{f}$ pulse shaper. The power at the sample was approximately $4 \mu \mathrm{J} /$ pulse for the visible and $2 \mu \mathrm{J} /$ pulse for the mid-IR, and the $1 / \mathrm{e}$ focal spot size radius was approximately $100 \mu \mathrm{m}$. The visible and IR beam angles were approximately $65^{\circ}$ and $56^{\circ}$ from the normal. Spectra were collected in the ssp polarization combination in which the IR was p-polarized, visible s-polarized, and the VSFG signal was s-polarized. 
PTCDI is generally considered an n-type organic semiconductor, which makes these devices susceptible to oxidation from environmental oxygen and water. A vacuum cell suitable for placement in the VSFG spectrometer was designed and at the University of Minnesota. The cell includes windows for input and generated beams and three vacuum ports: one for actively pumping on, a second for an electrical feed-through to make connections to OFETs under study, and a third for a vacuum pressure gauge. ${ }^{3}$ This allowed for applied biases to OFETs with in-situ VSFG studies under high vacuum.

VSFG spectra were averaged over a series of five exposures of 4 min each with a 4 min background acquired with the mid-IR blocked. Spectra were collected on transistor devices at the following gate voltages in order: $0,-80,-10,0,10,20,30,40,50,80,100,0,-80,0 \mathrm{~V}$. 


\section{Details of the fitting procedure}

The raw data were modeled at the electric field level and accounted for thin film interference effects, a non-resonant background, and four total Lorentzian resonances for the asymmetric $\left(v_{\mathrm{as}}\right)$ and symmetric $\left(v_{\mathrm{s}}\right)$ stretches at the inner and outer interfaces. ${ }^{4-5}$ The calculated intensity was given by the absolute value squared of the sum of the VSFG electric fields ( $E_{v, 0}^{\mathrm{VSFG}}$ ) coming from the $v_{\text {as }}$ and $v_{\text {as }}$ modes from either the air/PTCDI interface $(v=1)$ or the PTCDI/ $\mathrm{SiO}_{2}$ interface $(\mathrm{v}=2)$ :

$$
I_{0}^{\mathrm{ssp}} \propto\left|\sum_{v=1}^{2} E_{v, 0}^{\mathrm{VSFG}}\right|^{2}
$$

In the current work, only the ssp polarization combination was examined so no other combinations were modeled. In this case, $E_{v, 0}^{\mathrm{VSFG}}$ can be modeled as a sum of the resonant second-order susceptibilities from the asymmetric and symmetric stretches from either interface ( $\left.\chi_{v, q}^{(2), y y z}\right)$ multiplied by a transfer coefficient $T_{v}^{\mathrm{yyz}}$ for that interface $v$. The ssp combination samples the yyz component of $\chi^{(2)}$ when the frame is taken to have $\mathrm{x}$ and $\mathrm{y}$ in the sample plane.

$$
I_{0}^{s s p}=\left|T_{1}^{\mathrm{yyz}}\left(\chi_{1, \mathrm{as}}^{(2), \mathrm{yz}}+\chi_{1, \mathrm{~s}}^{(2), \mathrm{yyz}}\right)+T_{2}^{\mathrm{yyz}}\left(\chi_{2, \mathrm{as}}^{(2), \mathrm{yyz}}+\chi_{2, \mathrm{~s}}^{(2), \mathrm{yyz}}\right)+\chi_{\mathrm{NR}}^{(2)}\right|^{2}
$$

The transfer product $T_{v}^{\mathrm{yyz}}$ takes into account the reflection, refraction, and transmission of the incoming visible and IR fields and the outgoing VSFG field as they propagate through the entire thin film stack.

The second order susceptibility $\chi_{v, q}^{(2), y y z}$ represents the vibrationally resonant portion of the VSFG signal in which the structural information is located. It was modeled as a Lorentzian oscillator: 


$$
\chi_{v, q}^{(2)}=\frac{A_{v, q}}{\omega_{v, q}-\omega_{m I R}-i \Gamma_{v, q}}
$$

$A_{v, q}$ is the amplitude of the Lorentizian oscillator, $\Gamma_{v, q}$ is the oscillator HWHM=, $\omega_{v, q}$ is the resonant frequency, and $\omega_{m I R}$ is the frequency of the infrared light. The nonresonant susceptibility $\chi_{\mathrm{NR}}^{(2)}$ does not vary with IR frequency but is complex and can be expressed in polar form as:

$$
\chi_{\mathrm{NR}}^{(2)}=A_{\mathrm{NR}} e^{-i \phi}
$$

in which $A_{\mathrm{NR}}$ and $\phi$ are the nonresonant amplitude and phase. The nonresonant signal is treated as an effective susceptibility that has absorbed the effects of thin film interferences because the source of the nonresonant response is not well-characterized.

In fitting the spectra, the $T_{v}^{\mathrm{yyz}}$ were known from previous work ${ }^{4-5}$ and the $\chi_{v, q}^{(2), y y z}$ were parameterized to fit the data as follows. All of the VSFG spectra in Figure 2 were fitted simultaneously with the frequencies, widths, and amplitudes for the outer interface symmetric and asymmetric carbonyl stretches shared at all potentials but allowed to float. The inner interface frequencies and amplitudes for both modes were adjusted independently for all 8 gate potentials. The nonresonant magnitude and phase were also adjusted independently. The relative phases for inner and outer contributions of the same mode were kept opposite one another as well as the relative phase between the symmetric and asymmetric stretch on the same interface as described previously. ${ }^{4-7}$ The same peak width was used for the asymmetric and symmetric stretches at both interfaces but allowed to float. Tables S1 and S2 show the best fit results of this analysis. 
Table S1. Best fit parameters for asymmetric stretch $\left(v_{\mathrm{as}}\right)$ and non-resonant background from global fit to VSFG spectra.

\begin{tabular}{|c|c|c|c|c|c|c|c|}
\hline \multirow{2}{*}{$\begin{array}{c}\text { gate bias } \\
\text { (V) }\end{array}$} & \multicolumn{2}{|c|}{$v_{\text {as }}\left(\mathrm{cm}^{-1}\right)$} & \multicolumn{2}{|c|}{$A_{\text {as }} \times 10^{4}$} & \multirow{2}{*}{$\Gamma\left(\mathrm{cm}^{-1}\right)$} & \multirow{2}{*}{$A_{\mathrm{NR}}$} & \multirow{2}{*}{$\begin{array}{c}\phi_{\mathrm{NR}} \\
(\mathbf{r a d})\end{array}$} \\
\hline & outer & inner & outer & inner & & & \\
\hline 0 & & 1681.43 & & 5.702 & & 0.3174 & 1.1439 \\
\hline 10 & & 1681.01 & & 5.383 & & 0.3567 & 1.1270 \\
\hline 20 & & 1681.21 & & 4.515 & & 0.4108 & 1.0860 \\
\hline 30 & & 1680.77 & & 3.898 & & 0.4706 & 1.1436 \\
\hline & 1656.41 & & 2.886 & & 3.94 & & \\
\hline 40 & & 1679.09 & & 3.378 & & 0.5341 & 1.3517 \\
\hline 50 & & 1678.94 & & 3.323 & & 0.5921 & 1.3804 \\
\hline 80 & & 1678.98 & & 2.571 & & 0.7877 & 1.3868 \\
\hline 100 & & 1678.92 & & 2.323 & & 0.9241 & 1.4107 \\
\hline
\end{tabular}

\section{Asymmetric Stretching Region}

The asymmetric stretching region $\left(1650\right.$ to $\left.1680 \mathrm{~cm}^{-1}\right)$ was fit to two resonances for PTCDI at the outer and inner interfaces. Although it was fit in the same way as the symmetric region, the uncertainty from the low signal makes any in-depth analysis difficult. However, it is important to note that the $v_{\text {as }}$ resonances affect the overall fit of the data as they interfere with the nonresonant and symmetric stretch at the electric field level. The outer interface frequency and amplitude were shared at all potentials and fit to $1656.4 \mathrm{~cm}^{-1}$ and approximately 0.0003 . The inner frequencies and amplitudes were different for every potential; however, the frequency was found to be around $1680 \mathrm{~cm}^{-1}$ under all conditions. Figure S1 shows the center frequency of the inner $v_{\text {as }}$ as a function of applied potential demonstrating its gradual decrease with voltage. Over 
this voltage range, the amplitude decreases by about a factor of three. Comparing Table S1 to Table S2, the amplitudes of the asymmetric stretch were fit to be an order of magnitude smaller than the symmetric stretch. Also, the inner and outer $v_{\text {as }}$ are separated by $20 \mathrm{~cm}^{-1}$, which is much more than might be expected. There is certainly a fair amount of error on these frequency values given the signal-to-noise of the spectra in this region.

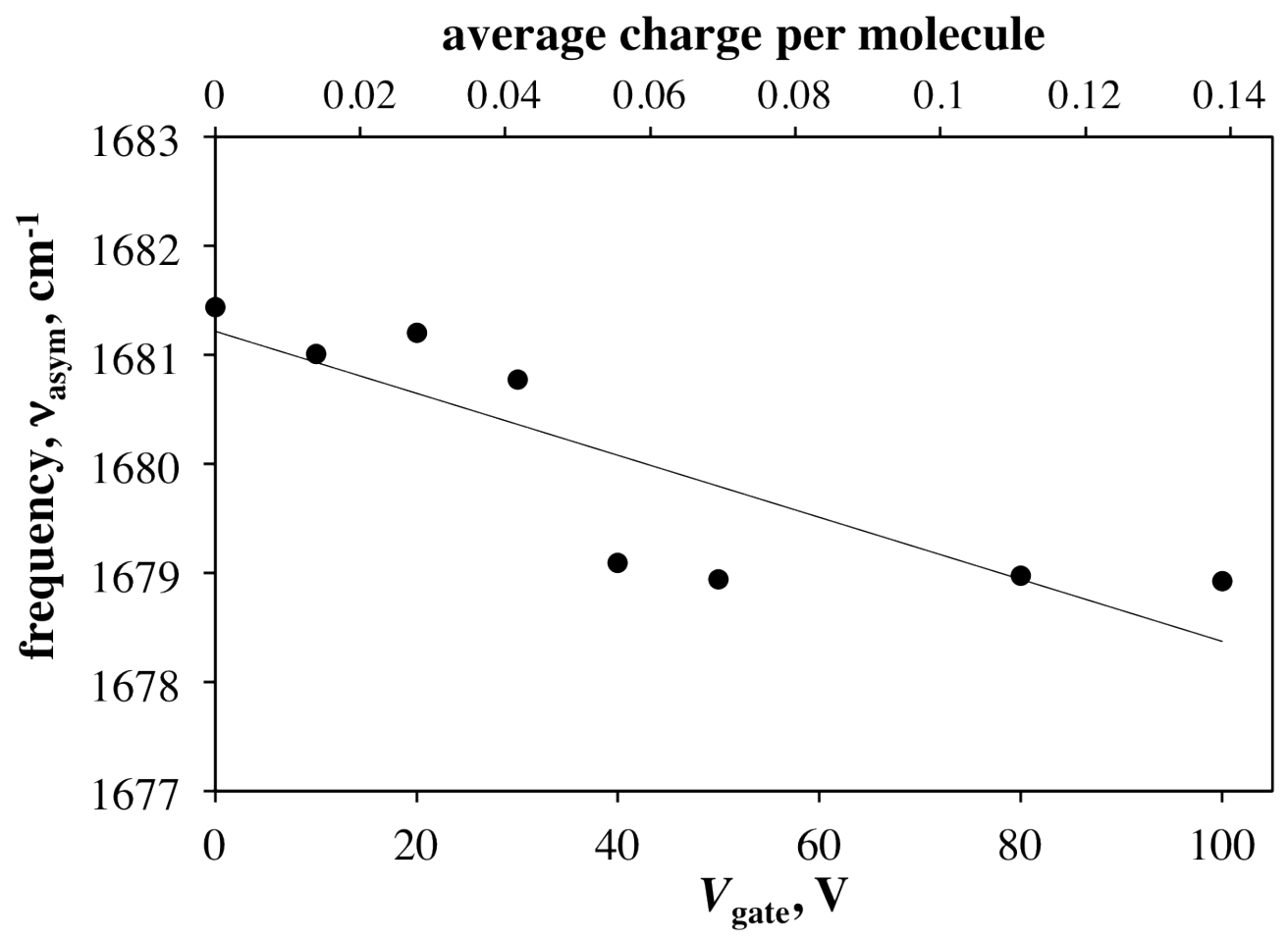

Figure S1. Best fit parameters for the inner (buried) interface imide asymmetric stretching frequency as a function of applied gate bias. Note that the average charge per molecule was derived as described in the main text, using the $v_{\mathrm{s}}$ mode rather than the $v_{\mathrm{as}}$.

\section{Vibrationally Non-resonant Signal}

The magnitude of the nonresonant signal increases linearly ( slope $=0.0062 \mathrm{~V}^{-1}$ ) as potential is increased (see Figure S2). This is expected as a portion of the nonresonant signal is from the electric field created at the dielectric surface and PTCDI surface that is increasing due to charge 
accumulation. ${ }^{2,8}$ The nonresonant susceptibility is predicted to increase linearly with the DC electric field strength. ${ }^{2,8}$ The nonresonant phase showed neither large changes nor a correlation with applied gate bias. Previous work showed that the phase of the nonresonant signal will undergo a rapid $\pi$ phase shift as the polarity of the applied potential is changed. ${ }^{2}$ However, in this work the polarity was always positive, therefore we would not expect any large changes in the direction of the interfacial electric field.

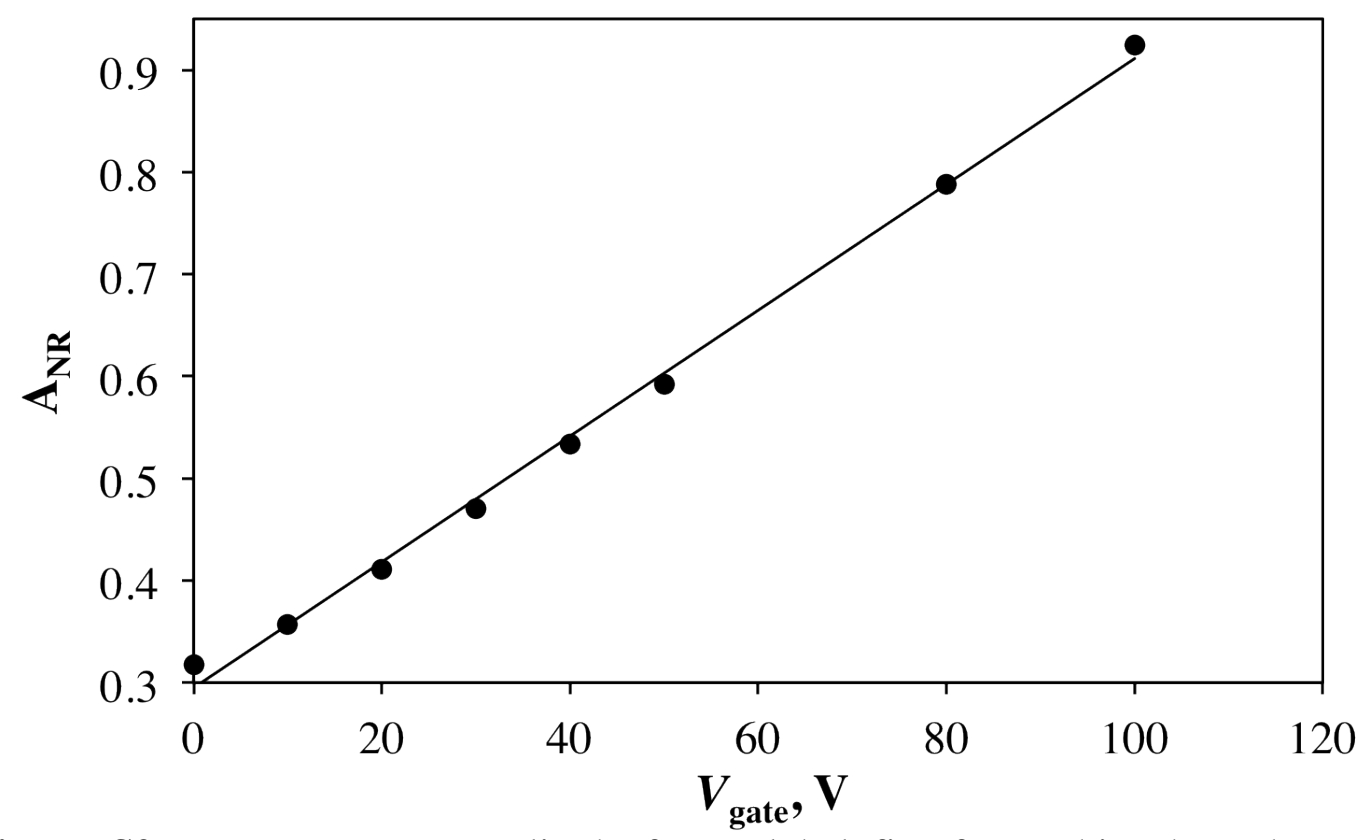

Figure S2. Non-resonant amplitude from global fit of gate bias-dependent VSFG spectra increases linearly due to charge accumulation at the buried interface.

\section{Symmetric Stretching Region}

In the case of the symmetric stretching mode, while the observed VSFG signal of the $v_{\mathrm{s}}$ region appears to increase as potential is increased, the actual value for the buried amplitude decreased as potential was increased (see Table S2). This illustrates the effects that thin film interference can have on the observed signal versus the actual strength of the interfacial oscillator. In this 
case, despite the fact that the observed signal strength of the symmetric stretch increased, the buried portion actually decreased.

Table S2. Best fit parameters for symmetric stretch $\left(v_{\mathrm{s}}\right)$ from global fit to VSFG spectra.

\begin{tabular}{|c|c|c|c|c|c|}
\hline \multirow{2}{*}{ gate bias (V) } & \multicolumn{2}{|c|}{$v_{\mathrm{s}}\left(\mathrm{cm}^{-1}\right)$} & \multicolumn{2}{|c|}{$A_{\mathrm{s}} \times 10^{3}$} & \multirow{2}{*}{$\Gamma\left(\mathrm{cm}^{-1}\right)$} \\
\hline & outer & inner & outer & inner & \\
\hline 0 & \multirow{8}{*}{1692.31} & 1693.49 & \multirow{8}{*}{2.54} & 2.99 & \multirow{8}{*}{3.94} \\
\hline 10 & & 1693.18 & & 2.74 & \\
\hline 20 & & 1692.46 & & 2.66 & \\
\hline 30 & & 1691.70 & & 2.62 & \\
\hline 40 & & 1691.17 & & 2.50 & \\
\hline 50 & & 1690.71 & & 2.13 & \\
\hline 80 & & 1689.11 & & 1.81 & \\
\hline 100 & & 1688.19 & & 1.63 & \\
\hline
\end{tabular}

The apparent increase in the amplitude of the symmetric stretch is actually a result of the shift in frequency of the buried interface as well as interference with the increasing nonresonant contribution. The resonances of the inner and outer $v_{\mathrm{s}}$ were fit to similar amplitudes. The frequency of the buried symmetric stretch red-shifts as the gate potential is increased. As the inner contribution of the symmetric stretch shifts farther from the position of the outer contribution they interfere with each other and with the nonresonant background more constructively, resulting in an overall increased resonant signal.

Typically, a change in the VSFG intensity of vibrational mode can be related to either a change in interfacial order or orientation if there is a corresponding increase in the output from another polarization combination. Other polarization combinations were not analyzed here so it can only be said that the decrease is likely caused by some kind of structural change at the interface that increases with potential. 


\section{Additional Details for DFT Calculations}

Density functional theory calculations were performed on various substituted PTCDI molecules in Gaussian 09 using the B3LYP functional and 6-311++g (d, p) basis set. Geometry optimizations were performed at lower basis sets and slowly built up to the 6-311++g (d,p) level of theory. ${ }^{9}$ The IR frequencies were calculated at the same level of theory as the geometry optimizations. CM5 population analyses of the molecules were also performed on the "core" of the molecule, as defined in Table S3..$^{10-13}$ The charges on the substituents attached to the nitrogen were ignored and the total charge on just the PTCDI core was determined. The results were then plotted on a graph of frequency vs. excess charge on the core.

The substituents of PTCDI were aliphatic and a nitrile. These substituents were chosen to span a range of fractional charges on the core from 0 to -1 since PTCDI undergoes n-type transport during OFET operation. Substituents were chosen to be similar to the alkyl side chains in the PTCDI- $\mathrm{C}_{8}$ used in the experimental work in this study. The resulting carbonyl stretching vibrations in the substituted PTCDIs were visualized to verify there was negligible coupling with vibrations in the chosen substituents. This was done to ensure that the change in fractional charge on the PTCDI core was due solely to the donation of electron density to the core as a result of the substituent and not due to geometric or coupling effects. Chains containing F, O, $\mathrm{OH}$ substituents and $\mathrm{N}$ were tested but excluded for this reason. In those cases, changes in the total charge of the molecule would lead to charge changes on the perylene core but would not change the frequency of the carbonyl vibration.

Table S3 shows the calculated $v_{\mathrm{s}}$ frequencies (scaled by a factor of 0.97$)^{14}$ for a variety of substituted PTCDI species. Also included are experimental frequencies for dimethyl-PTCDI, ${ }^{15}$ unsubstituted PTCDI, ${ }^{16}$ adamantylthio-substituted PTCDI,${ }^{17}$ and PTCDI- $\mathrm{C}_{8}$ anion and neutral 
forms. ${ }^{18}$ These data were plotted in Figure 4 , and the core charges for the experimental frequencies were taken to be the same as the calculated values for the same molecule. An exception was the adamantylthio-substituted PTCDI, which did not converge computationally and was simply assigned core charge values of $0,-1$, and -2 . The calculated frequencies are in good agreement with experimental results.

The data were fit to a straight line. Work by others had shown linear behavior for vibrational frequencies with fractional charge. ${ }^{19}$ As electrons are either added to the anti-bonding LUMO or removed from the HOMO of the PTCDI core, they will affect the frequency of the bond in a proportional way. On initial examination this seems to hold, the nitrile group leads to a fractional charge of -0.0251 and resonance for the carbonyl symmetric stretch of $1696 \mathrm{~cm}^{-1}$ while the more electron donating ethyl group leads to a charge of -0.444 and a frequency $1687 \mathrm{~cm}^{-1}$. The ethyl and acetyl groups are less electron donating with charges of -0.282 and -0.1825 , but their frequencies do not follow this trend with the ethyl substituted and acetyl substituted PTCDIs having resonances at $1697 \mathrm{~cm}^{-1}$ and $1666 \mathrm{~cm}^{-1}$, respectively. However, when ethyl functionalized PTCDI is ionized into anion or cationic form the overall trend is maintained with the charges and frequency for cationic, neutral and anionic ethyl substituted PTCDI being: 0.584 and $1705 \mathrm{~cm}^{-1},-0.282$ and $1697 \mathrm{~cm}^{-1}$, and -1.146 and $1652 \mathrm{~cm}^{-1}$, respectively. The results for acetyl substituted PTCDI seem to not follow the trend with: 0.712 and $1724 \mathrm{~cm}^{-1},-0.183$ and $1666 \mathrm{~cm}^{-1}$, and -1.06 and $1667 \mathrm{~cm}^{-1}$. Between the neutral and anionic forms the trend breaks down for the acetyl substituted PTCDI. This could mean there is something other than charge density on the PTCDI core effecting frequency of the carbonyl bond. Notwithstanding these uncertainties, there is still a strong linear trend of the overall data (see Figure 4). The data were 
fitted to a line with a slope of $39.2 \mathrm{~cm}^{-1} /$ charge and a $y$-intercept of $1694 \mathrm{~cm}^{-1}$ with an R-squared value of 0.86 . 
Table S3. Best fit parameters for symmetric stretch $\left(v_{\mathrm{s}}\right)$ from global fit to VSFG spectra.

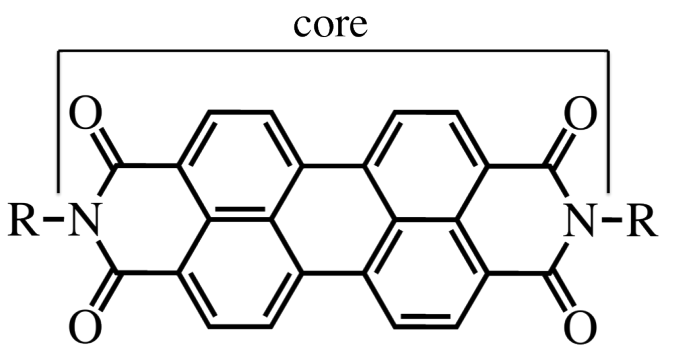

\begin{tabular}{|c|c|c|c|c|}
\hline$\cdots \cdot \mathrm{R}$ & charge type & $\begin{array}{c}\text { calculated } \\
v_{\mathrm{s}}\left(\mathrm{cm}^{-1}\right)\end{array}$ & charge on core & $\begin{array}{c}\text { experimental } \\
v_{\mathrm{s}}\left(\mathrm{cm}^{-1}\right)\end{array}$ \\
\hline & double anion & & -2 & $1606^{17}$ \\
\hline & anion & 1646.09 & -1 & $1642^{17}$ \\
\hline$\cdots \cdot \cdot \mathrm{H}$ & neutral & 1697.50 & 0 & $\begin{array}{l}1688^{16} \\
1696^{17}\end{array}$ \\
\hline & cation & 1714.96 & 1 & \\
\hline & anion & 1634.45 & -1.36 & \\
\hline$\cdots \cdot \mathrm{CH}_{3}$ & neutral & 1688.29 & -0.45 & $1692^{15}$ \\
\hline & cation & 1703.81 & 0.46 & \\
\hline & anion & 1633.48 & -1.33 & \\
\hline $\mathrm{CH}_{3}$ & neutral & 1687.32 & -0.44 & \\
\hline & cation & 1699.93 & 0.46 & \\
\hline & neutral & 1687.32 & -0.43 & \\
\hline $\mathrm{H}_{3}$ & anion & 1633.00 & -1.32 & \\
\hline & anion & 1652.88 & -1.15 & \\
\hline$\therefore \nabla_{\mathrm{CH}_{2}}$ & neutral & 1697.99 & -0.28 & \\
\hline & cation & 1705.26 & 0.58 & \\
\hline$\cdots \cdot \mathrm{C}_{0} \mathrm{H}$ & anion & & -1.32 & $1629^{18}$ \\
\hline-8117 & neutral & & -0.43 & $1696^{18}$ \\
\hline & anion & 1667.43 & -1.1 & \\
\hline$\cdots \cdot \mathrm{C} \equiv \mathrm{CH}$ & neutral & 1666.46 & -0.18 & \\
\hline & cation & 1724.66 & 0.71 & \\
\hline & anion & 1680.04 & -0.90 & \\
\hline$\cdots \cdot \mathrm{C} \equiv \mathrm{N}$ & neutral & 1696.05 & -0.03 & \\
\hline & cation & 1741.64 & 0.90 & \\
\hline
\end{tabular}




\section{REFERENCES}

1. O'Brien, D. B.; Anglin, T. C.; Massari, A. M. Surface Chemistry and Annealing-Driven Interfacial Changes in Organic Semiconducting Thin Films on Silica Surfaces. Langmuir 2011, 27, 13940-13949.

2. Anglin, T. C.; O'Brien, D. B.; Massari, A. M. Monitoring the Charge Accumulation Process in Polymeric Field-Effect Transistors Via in Situ Sum Frequency Generation. J. Phys. Chem. C 2010, 114, 17629-17637.

3. O'Brien, D. B. Solving the Two-Interface Problem in Vibrational Sum Frequency Generation Spectroscopy Applied to Multilayer Thin Film Systems. University of Minnesota Twin Cities, Minneapolis, 2014.

4. O'Brien, D. B.; Massari, A. M. Simulated Vibrational Sum Frequency Generation from a Multilayer Thin Film System with Two Active Interfaces. J. Chem. Phys. 2013, 138, 154708.

5. O'Brien, D. B.; Massari, A. M. Modeling Multilayer Thin Film Interference Effects in Interface-Specific Coherent Nonlinear Optical Spectroscopies. J. Opt. Soc. Am. B 2013, 30, 1503-1512.

6. O'Brien, D. B.; Massari, A. M. Experimental Evidence for an Optical Interference Model for Vibrational Sum Frequency Generation on Multilayer Organic Thin Film Systems. I. Electric Dipole Approximation. J. Chem. Phys. 2015, 142, 024703.

7. O'Brien, D. B.; Massari, A. M. Experimental Evidence for an Optical Interference Model for Vibrational Sum Frequency Generation on Multilayer Organic Thin Film Systems. Ii. Consideration for Higher Order Terms. J. Chem. Phys. 2015, 142, 024704. 
8. Ong, S. W.; Zhao, X. L.; Eisenthal, K. B. Polarization of Water-Molecules at a Charged Interface - 2nd Harmonic Studies of the Silica Water Interface. Chem. Phys. Lett. 1992, 191, 327-335.

9. Frisch, M. J.; Trucks, G. W.; Schlegel, H. B.; Scuseria, G. E.; Robb, M. A.; Cheeseman, J. R.; Scalmani, G.; Barone, V.; Mennucci, B.; Petersson, G. A., et al. Gaussian 09 Revision D.01, Gaussian, Inc.: Wallingford, CT, USA, 2009.

10. Hirshfeld, F. L. Bonded-Atom Fragments for Describing Molecular Charge-Densities. Theor. Chim. Acta 1977, 44, 129-138.

11. Marenich, A. V.; Jerome, S. V.; Cramer, C. J.; Truhlar, D. G. Charge Model 5: An Extension of Hirshfeld Population Analysis for the Accurate Description of Molecular Interactions in Gaseous and Condensed Phases. J. Chem. Theory Comput. 2012, 8, 527-541.

12. Ritchie, J. P. Electron-Density Distribution Analysis for Nitromethane, Nitromethide, and Nitramide. J. Am. Chem. Soc. 1985, 107, 1829-1837.

13. Ritchie, J. P.; Bachrach, S. M. Some Methods and Applications of Electron-Density Distribution Analysis. J. Comput. Chem. 1987, 8, 499-509.

14. Andersson, M. P.; Uvdal, P. New Scale Factors for Harmonic Vibrational Frequencies Using the B3LYP Density Functional Method with the Triple-Xi Basis Set 6-311+G(D,P). J. Phys. Chem. A 2005, 109, 2937-2941.

15. Friedrich, M.; Gavrila, G.; Himcinschi, C.; Kampen, T. U.; Kobitski, A. Y.; Mendez, H.; Salvan, G.; Cerrillo, I.; Mendez, J.; Nicoara, N., et al. Optical Properties and Molecular Orientation in Organic Thin Films. J. Phys.-Condens. Mat. 2003, 15, S2699-S2718. 
16. Chis, V.; Mile, G.; Stiufiuc, R.; Leopold, N.; Oltean, M. Vibrational and Electronic Structure of Ptcdi and Melamine-Ptcdi Complexes. J. Mol. Struct. 2009, 924-26, 47-53.

17. Slater, A. G.; Davies, E. S.; Argent, S. P.; Lewis, W.; Blake, A. J.; McMaster, J.; Champness, N. R. Bis-Thioether-Substituted Perylene Diimides: Structural, Electrochemical, and Spectroelectrochemical Properties. J. Org. Chem. 2013, 78, 2853-2862.

18. Kaake, L. G.; Zou, Y.; Panzer, M. J.; Frisbie, C. D.; Zhu, X. Vibrational Spectroscopy Reveals Electrostatic and Electrochemical Doping in Organic Thin Film Transistors Gated with a Polymer Electrolyte Dielectric. J. Am. Chem. Soc. 2007, 129, 7824-7830.

19. Nanova, D.; Beck, S.; Fuchs, A.; Glaser, T.; Lennartz, C.; Kowalsky, W.; Pucci, A.; Kroeger, M. Charge Transfer in Thin Films of Donor-Acceptor Complexes Studied by Infrared Spectroscopy. Org. Electron. 2012, 13, 1237-1244. 